\title{
Mask intermittent positive pressure ventilation in chronic hypercapnic respiratory failure due to chronic obstructive pulmonary disease
}

\author{
P. Sivasothy, I.E. Smith, J.M. Shneerson
}

Mask intermittent positive pressure ventilation in chronic hypercapnic respiratory failure due to chronic obstructive pulmonary disease. P. Sivasothy, I.E. Smith, J.M. Shneerson. CERS Journals Ltd 1998.

ABSTRACT: Noninvasive ventilation in chronic obstructive pulmonary disease (COPD) has been shown to improve arterial blood gases but its long-term role has not been established.

We retrospectively studied 26 consecutive patients with hypercapnic ventilatory failure due to COPD in whom oxygen therapy caused worsening hypercapnia (defined as a rise in the daytime arterial carbon dioxide tension $\left(\mathrm{Pa}_{1}, \mathrm{CO}_{2}\right)$ to $>8.0 \mathrm{kPa}$ or nocturnal transcutaneous carbon dioxide tension $\left(P_{t c}, \mathrm{CO}_{2}\right)$ to $\left.>9 \mathrm{kPa}\right)$. All were treated with mask ventilation (15 with nasal and 11 face masks) at night and during daytime naps. Additional oxygen therapy was required in 15 patients.

The mean annualized death rate was $10.8 \%$ with a 1 and 3 yr survival of 92 and $68 \%$, respectively. After $1 \mathrm{yr}$ the median daytime $\mathrm{Pa}_{\mathrm{a}} \mathrm{CO}_{2}$ had fallen by $1.35 \mathrm{kPa}$ and the arterial oxygen tension $\left(\mathrm{Pa}, \mathrm{O}_{2}\right)$ had risen by $2.4 \mathrm{kPa}$. In subjects not using additional oxygen the median overnight $\mathrm{Sa}_{\mathrm{a}} \mathrm{O}_{2}$ rose by $12 \%$ and the $\mathrm{Ptc}_{\mathrm{tc}} \mathrm{CO}_{2}$ fell by $2.8 \mathrm{kPa}$ after $1 \mathrm{yr}$. The haematocrit was significantly less than pretreatment at 6 months and 1 yr. Quality of life in the domain of role limitation by physical health (measured using the SF-36 questionnaire) improved significantly at 6 months.

Survival in this selected group with clinically stable airflow obstruction unable to tolerate oxygen therapy and treated with noninvasive mask ventilation is better than historical controls and is comparable to those able to tolerate oxygen therapy. Poor survival was associated with a low forced expiratory volume in one second, a low body mass index and a high nocturnal transcutaneous carbon dioxide tension. No difference in survival was found between those treated with mask intermittent positive pressure ventilation alone or with mask intermittent positive pressure and supplementary oxygen therapy.

Eur Respir J 1998; 11: 34-40.

The use of noninvasive ventilation in pulmonary disease has been shown to improve the blood gases of pati-ents both while awake [1-3] and asleep [1, 4, 5] but it has proved difficult to identify in which patients survival or quality of life can be improved [5-7]. Mask intermittent positive pressure ventilation (MIPPV) has been introduced in acute respiratory failure in patients with chronic obstructive pulmonary disease (COPD) as a short-term measure to reduce intubation rates and improve survival [8].

Long-term oxygen therapy (LTOT) improves survival in chronically hypoxic COPD patients [9, 10]; however, patients with a high arterial carbon dioxide tension $\left(\mathrm{Pa}_{\mathrm{a}} \mathrm{CO}_{2}\right)$ had a poor prognosis both prior to oxygen therapy $[11,12]$ and (at least in the first year) with oxygen therapy [13]. In some patients, hypercapnia deteriorates with oxygen therapy manifesting with headaches, confusion, drowsiness and difficulty concentrating such that LTOT is not tolerated. Long-term MIPPV could in principle prevent this complication and improve survival.

We report our experience with nocturnal mask ventilation in patients with COPD with hypercapnic respiratory
Respiratory Support and Sleep Centre, Papworth Hospital, Papworth Everard, Cambridge, UK.

Correspondence: P. Sivasothy

Respiratory Support and Sleep Centre

Papworth Hospital

Papworth Everard

Cambridge CB3 8RE

UK

Fax: 00441480831315

Keywords: Chronic obstructive pulmonary disease

hypercapnic respiratory failure noninvasive mechanical ventilation

Received: March 31997

Accepted after revision November 71997 failure in whom oxygen therapy resulted in worsening daytime or nocturnal hypercapnia.

\section{Materials and methods}

\section{Patient selection}

The inclusion criteria were: 1) arterial oxygen tension $\left(P \mathrm{a}, \mathrm{O}_{2}\right)<7.3 \mathrm{kPa}$ and $\mathrm{Pa}_{\mathrm{a}} \mathrm{CO}_{2}>6.0 \mathrm{kPa}$ while breathing room air; 2) stable clinical state, arterial blood gases and pulmonary function tests over the 3 weeks prior to the study (with a daytime arterial $\mathrm{H}^{+}$concentration between $\left.35-48 \mathrm{nmol}^{-1}\right)$; and 3 ) supplementary oxygen therapy failed either to raise the daytime $\mathrm{Pa}, \mathrm{O}_{2}$ to $>7.3 \mathrm{kPa}$ without a rise in the awake $P \mathrm{a}_{1} \mathrm{CO}_{2}$ to $>8 \mathrm{kPa}$ or failed to raise nocturnal oxygen saturations to $>90 \%$ without a rise in the transcutaneous carbon dioxide tension $\left(P_{\mathrm{tc}}, \mathrm{CO}_{2}\right)$ to $>9 \mathrm{kPa}$. Patients with co-existing pulmonary diseases, neuromuscular or chest wall disorders were excluded. Twenty six patients, 13 males and 13 females, fulfilling these criteria were studied retrospectively. 


\section{Methods}

All patients had initial arterial blood gas measurements (Ciba-Corning 238 Blood gas analyser; Medfield, MA, USA) performed breathing room air. Patients had haemoglobin concentration and haematocrit assessed at presentation. Total lung capacity (TLC) was assessed by body plethysmography (Masterlab, Jaegar, Wurzburg, Germany), forced expiratory volume in one second (FEV1) and forced vital capacity (FVC) with a rolling seal spirometer (Vitalograph, Buckingham, UK) and the transfer factor of the lung for carbon monoxide (TL,CO) with a gas analyser (TT Autolink; P.K. Morgan, Rainham, UK).

Hypercapnia was assessed by continuous $P \mathrm{tc}, \mathrm{CO}_{2}$ monitoring (Radiometer TCM 3; Copenhagen, Denmark) and $P a, \mathrm{CO}_{2}$. Oxygenation was assessed using continuous oxygen saturation $\left(\mathrm{Sa}_{\mathrm{a}} \mathrm{O}_{2}\right)$ monitored by a pulse oximeter (Ohmeda Biox 3740; Louisville, CO, USA) and $\mathrm{Pa}_{\mathrm{a}} \mathrm{O}_{2}$. Data from both the pulse oximeter and transcutaneous capnometer were simultaneously recorded on a chart recorder (Dash 3; Astromed, Warwick, RI, USA). Recordings were made as supplementary oxygen was titrated in increments of no more than $0.25 \mathrm{~L} \cdot \mathrm{min}^{-1}$, to raise the oxygen saturation to $>90 \%$.

All patients were established on noninvasive MIPPV which was either with the volume preset Monnal D ventilator (TAEMA, Paris, France) or a pressure preset ventilator, either Nippy (Friday Medical Ltd, London, UK) or bi-level positive airway pressure (BiPAP) (Respironics Inc, Murraysville, PA, USA). MIPPV was initiated during an in-patient episode in each case. Oxygen therapy was given on initiation of MIPPV or subsequent review of patients to increase night-time and daytime $\mathrm{Sa}_{\mathrm{a}} \mathrm{O}_{2}$ to $>90 \%$ if this could not be achieved despite a reduction of the $P \mathrm{a}, \mathrm{CO}_{2}$ with MIPPV.

Patients were reviewed at 1 month, 6 months, 12 months, and 2 yrs. At each review, daytime arterial gas, overnight $P \mathrm{tc}, \mathrm{CO}_{2}$ and $\mathrm{Sa}_{\mathrm{a}} \mathrm{O}_{2}$, haemoglobin and haematocrit measurements were made. Patient ventilator settings and oxygen therapy were adjusted according to these results. Data obtained on patients who had an infective exacerbation of their pulmonary disease were excluded from later analysis at that time point. An infective exacerbation was defined as cough productive of mucopurulent or purulent sputum with either new changes on a chest radiograph or a raised white blood cell count.

Quality of life was assessed in 11 patients who were studied prior to MIPPV and after 1, 6 and 12 months of treatment using the SF-36 questionnaire. This measures the patient's perceptions of health in the domains of physical functioning, role limitations because of physical problems, bodily pain, general health perceptions, vitality, social functioning, role limitation because of emotional problems and mental health.

\section{Statistical analysis}

Statistical analysis of all data was performed using the Statistical Products and Service Solutions (SPSS) software for Windows (SPSS; Chicago, IL, USA). Comparisons of results between review visits was made using the Wilcoxon matched pairs signed rank test. Arterial blood gas measurement, $\mathrm{Sa}_{\mathrm{a}} \mathrm{O}_{2}$ and $\mathrm{Ptc}, \mathrm{CO}_{2}$ at differing inspiratory oxygen fractions $\left(\mathrm{F} \mathrm{I}_{2} \mathrm{O}_{2}\right)$ on the same patient were used for paired analysis of $\mathrm{Pa}, \mathrm{CO}_{2}$ or $P \mathrm{tc}, \mathrm{CO}_{2}$ but not for comparisons of overnight mean $\mathrm{Sa}_{2} \mathrm{O}_{2}$. Survival was estimated using the Kaplan-Meier method from the initiation of MIPPV and survival comparisons were made using the log rank test between those using MIPPV alone or MIPPV with supplementary oxygen therapy. Analyses of predictive factors were made using the Mann-Whitney $U$ test looking between: 1) survivors and nonsurvivors; and 2) those using MIPPV alone or MIPPV with supplementary oxygen therapy with regard to the variables of age, smoking history, per cent predicted FEV1, body mass index (BMI), haematocrit and initial blood gases and blood gases at 1 month. Statistical significance was taken as $\mathrm{p}<0.05$ with the Bonferroni adjustment used to correct for repeat pairwise comparisons.

\section{Results}

The median age of patients at entry into the study was 66 (range 41-78) yrs. The median duration of follow-up was 17.5 (range 4-74) months. Self-reported smoking history, available in 25 patients, showed patients had been smoking for $43 \pm 25$ (mean \pm sD) pack-years. The mean \pm SD FEV1 was $0.68 \pm 0.37 \mathrm{~L}(27 \pm 14.6 \%$ pred $)$ and the mean \pm SD FVC was $1.99 \pm 0.49 \mathrm{~L}(63 \pm 17.8 \%$ pred $)$. The gas transfer was reduced with a TL,CO of $4.6 \pm 2.27 \mathrm{mmol} \cdot \mathrm{min}^{-1}$. $\mathrm{kPa}^{-1}(58 \pm 25.9 \%$ pred) and carbon monoxide transfer coefficient $(K \mathrm{CO}) 1.10 \pm 0.46 \mathrm{mmol} \cdot \mathrm{min}^{-1} \cdot \mathrm{kPa}^{-1} \cdot \mathrm{L}^{-1}$. TL,CO values were unavailable in seven patients due to insufficient volumes for single breath analysis. Arterial blood gas measurements at presentation were performed on air in all patients. Sixteen patients had a daytime $P \mathrm{a}, \mathrm{CO}_{2}>8 \mathrm{kPa}$ on daytime arterial measurement with supplementary oxygen therapy. The remaining 10 patients had a $P \mathrm{tc}, \mathrm{CO}_{2}>9 \mathrm{kPa}$ during a night-time study, with added oxygen.

Initially 13 patients were treated with MIPPV alone at night since it raised night-time $\mathrm{Sa}_{\mathrm{a}} \mathrm{O}_{2}$ to $>90 \%$. In the other 13 patients MIPPV was given with supplementary oxygen therapy initially at night but also during the day when their $P \mathrm{a}, \mathrm{CO}_{2}$ had been reduced. Subsequently supplementary oxygen therapy was introduced in two patients from the MIPPV alone group to maintain night-time $\mathrm{Sa}_{\mathrm{a}} \mathrm{O}_{2}>90 \%$. In patients prescribed oxygen, a combined total of at least $15 \mathrm{~h} \cdot \mathrm{day}^{-1}$ use of nocturnal ventilation and daytime oxygen was recommended. In 13 out of 15 patients started on oxygen therapy it was prescribed at a rate of $ð 1 \mathrm{~L} \cdot \mathrm{min}^{-1}$ and six were prescribed $0.5 \mathrm{~L} \cdot \mathrm{min}^{-1}$. During subsequent in-patient assessments the $F \mathrm{I}_{1} \mathrm{O}_{2}$ was increased to maintain $\mathrm{S}_{\mathrm{a}}, \mathrm{O}_{2}>$ $90 \%$ in five patients and decreased in two patients for hypercapnia. The mean duration of the initial stay was $10 \pm 2.3$ days.

All patients tolerated MIPPV either via nasal mask (15 patients) or full face mask (11 patients). Compliance judged by hours of measured ventilation per day, using the internal ventilator clock, was 7.81 \pm 1.61 (mean \pm SD). Six patients died after starting MIPPV at 4, 5, 13, 14, 17 and 22 months. In all six patients death occurred during a chest infection with acute respiratory failure. One patient had developed a bronchial carcinoma but had continued with MIPPV.

The mean \pm SEM survival time was $55.1 \pm 6.92$ months. Baseline comparisons between survivors and nonsurvivors with regard to age, $\mathrm{FEV} 1, P \mathrm{a}, \mathrm{CO}_{2}, \mathrm{~Pa}_{\mathrm{a}} \mathrm{O}_{2}, \mathrm{BMI}$, and 
Table 1. - A comparison of results between survivors and nonsurvivors at pretreatment and 1 month

\begin{tabular}{|c|c|c|}
\hline & $\begin{array}{l}\text { Survivors } \\
(\mathrm{n}=20)\end{array}$ & $\begin{array}{l}\text { Nonsurvivors } \\
\quad(\mathrm{n}=6)\end{array}$ \\
\hline Age yrs & $67.0 \quad(41-78.0)$ & $60.0(49.0-71.0)$ \\
\hline FEV1*\% pred & $26.0(14.8-64.3)$ & $15.2(7.7-18.5)$ \\
\hline$P \mathrm{a}, \mathrm{CO}_{2} \mathrm{kPa}$ & $8.2(6.5-9.6)$ & $9.6(7.2-11.8)$ \\
\hline$P \mathrm{a}, \mathrm{O}_{2} \mathrm{kPa}$ & $5.8(4.0-6.9)$ & $5.0 \quad(4.6-6.9)$ \\
\hline$P \mathrm{a}, \mathrm{CO}_{2}$ at 1 month $\mathrm{kPa}$ & $7.5(6.1-9.1)$ & $7.5 \quad(6.5-14.2)$ \\
\hline$P \mathrm{a}, \mathrm{O}_{2}$ at 1 month $\mathrm{kPa}$ & $7.5 \quad(6.1-10.3)$ & $7.0 \quad(5.5-8.5)$ \\
\hline $\mathrm{Sa}_{\mathrm{a}, \mathrm{O}_{2}}$ daytime $\%$ & $79.0(56.0-88.0)$ & $73.0(65.0-86.0)$ \\
\hline$P \mathrm{tc}, \mathrm{CO}_{2} * \mathrm{kPa}$ & $8.9 \quad(7.4-11.0)$ & $11.4 \quad(7.8-12.5)$ \\
\hline BMI* & $29.2(20.4-43.8)$ & $19.7(15.5-30.1)$ \\
\hline Duration of stay* days & $9.5(6.0-13.0)$ & $12.0(11.0-14.0)$ \\
\hline
\end{tabular}

Values are presented as median with range in parenthesis. $\mathrm{FEV} 1$ : forced expiratory volume in one second; $\mathrm{Pa}_{\mathrm{a}} \mathrm{CO}_{2}$ : arterial carbon dioxide tension; $\mathrm{Pa}_{\mathrm{a}} \mathrm{O}_{2}$ : arterial oxygen tension; $\mathrm{Sa}, \mathrm{O}_{2}$ : arterial oxygen saturation; $P_{\mathrm{tc}}, \mathrm{CO}_{2}$ : transcutaneous carbon dioxide tension; BMI: body mass index. *: $\mathrm{p}<0.05$.

Table 2. - A comparison of results between mask intermittent positive pressure ventilation (MIPPV) alone or with oxygen therapy at pretreatment and 1 month

\begin{tabular}{|c|c|c|}
\hline & $\begin{array}{c}\text { MIPPV + oxygen } \\
(\mathrm{n}=13)\end{array}$ & $\begin{array}{l}\text { MIPPV alone } \\
\quad(\mathrm{n}=13)\end{array}$ \\
\hline Age yrs & $65.0(41.0-77.0)$ & $71.0(54.0-78.0)$ \\
\hline FEV $1 \%$ pred & $20.0(7.7-44.2)$ & $23.5(15.2-64.3)$ \\
\hline$P \mathrm{a}, \mathrm{CO}_{2} \mathrm{kPa}$ & $7.9 \quad(6.5-11.8)$ & 8.8 (7.1-9.7) \\
\hline$P a, O_{2} \mathrm{kPa}$ & $5.9 \quad(4.0-6.9)$ & $5.7 \quad(4.7-6.9)$ \\
\hline$P \mathrm{a}, \mathrm{CO}_{2}$ at 1 month $\mathrm{kPa}$ & $7.5(6.1-14.2)$ & $7.45(6.5-8.4)$ \\
\hline${\mathrm{Pa}, \mathrm{O}_{2}}_{2}$ at 1 month $\mathrm{kPa}$ & $7.4 \quad(6.2-11.7)$ & $7.6 \quad(6.5-10.2)$ \\
\hline $\mathrm{Sa}_{\mathrm{a}, \mathrm{O}_{2}}$ daytime $\%$ & $79.0(56.0-88.0)$ & $78.0(66.0-87.0)$ \\
\hline$P_{\mathrm{tc}, \mathrm{CO}_{2}} \mathrm{kPa}$ & $10.0 \quad(7.4-12.5)$ & $8.7 \quad(7.6-11.0)$ \\
\hline BMI & $23.9(16.1-40.5)$ & $31.0(15.5-43.8)$ \\
\hline Duration of stay days & $11.0 \quad(9.0-14.0)$ & $(6-13)$ \\
\hline
\end{tabular}

Values are presented as median with range in parenthesis. No significant differences were demonstrated. For definitions see legend to table 1 .

duration of first stay (median and range) are shown in table 1 . The patients who died had a significantly lower FEV1 and BMI, a higher median overnight $P$ tc, $\mathrm{CO}_{2}$ and a longer duration of first hospital admission. Although $P \mathrm{a}, \mathrm{CO}_{2}$ was correlated with overnight $P \mathrm{tc}, \mathrm{CO}_{2}(\mathrm{p}<0.05)$ there was no association between it and survival. No significant differences between patients using MIPPV alone or those with supplementary oxygen therapy were shown for age, smoking duration, FEV1 or pretreatment arterial blood gas measurement (table 2). The Kaplan-Meier survival analysis with the log rank test showed no significant differences between the two groups (fig. 1).

There was a statistically significant fall $(\mathrm{p}<0.05)$ of daytime $P$ a, $\mathrm{CO}_{2}$ and rise in $P$ a, $\mathrm{O}_{2}$ at 1 and 6 months and 1 and 2 yrs compared to pretreatment (figs. 2 and 3 ). There were no significant changes when daytime $P \mathrm{a}, \mathrm{CO}_{2}$ and $\mathrm{Pa}, \mathrm{O}_{2}$ at 1 month were compared to those at 6 months, 1 and 2 yrs. Similarly overnight median $P \mathrm{tc}, \mathrm{CO}_{2}$ and $\mathrm{Sa}_{\mathrm{a}} \mathrm{O}_{2}$ showed statistically significant decreases and increases respectively at 1 and 6 months, and 1 and 2 yrs compared to pretreatment with no significant changes between 1 and 6 months, and 1 and 2 yrs (figs. 4 and 5). The successive median haematocrit estimates up to 2 yrs showed a continuing decrease compared to admission $(\mathrm{p}<0.05)$ (fig. 6). The

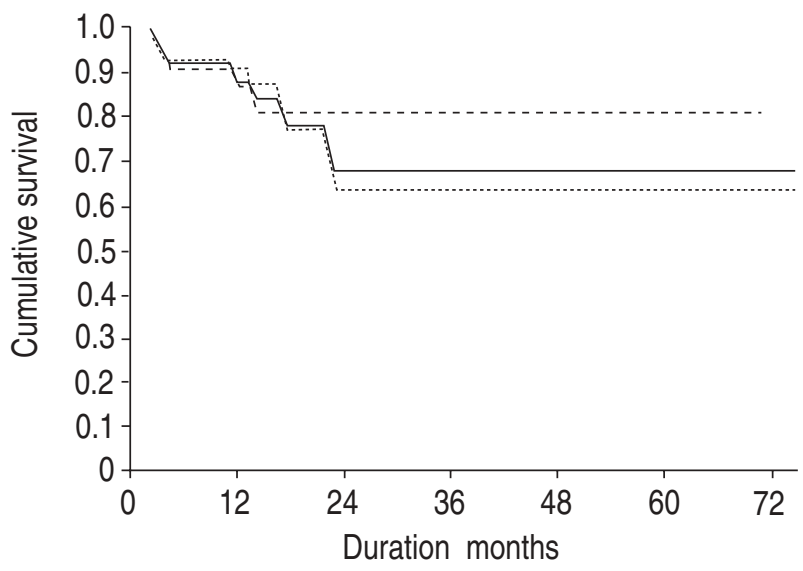

Fig. 1. - Kaplan-Meier survival graph for patients treated with mask intermittent positive pressure ventilation (MIPPV). _ _ : whole group; ...... : with oxygen; ------: without oxygen. Mean ( \pm SEM) survival was: for the whole group $55 \pm 6.9$ months; for the MIPPV group with supplementary oxygen therapy $53 \pm 8.9$ months; and for the MIPPV group alone $55 \pm 7.3$ months.

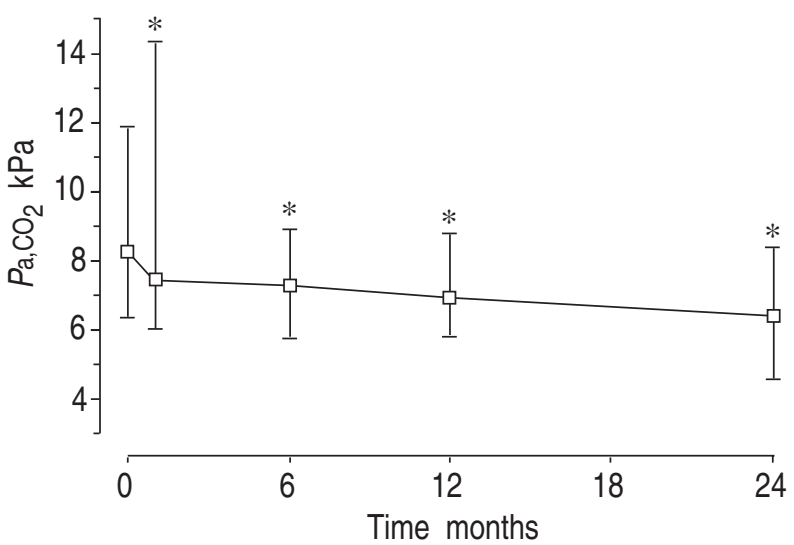

Fig. 2. - Median values and range in arterial carbon dioxide tensions $\left(P_{\mathrm{a}}, \mathrm{CO}_{2}\right)$ in patients treated with mask intermittent positive pressure ventilation at: pretreatment $(\mathrm{n}=26) ; 1$ month $(\mathrm{n}=20) ; 6$ months $(\mathrm{n}=19) ; 1 \mathrm{yr}$ $(\mathrm{n}=18)$; and 2 yrs $(\mathrm{n}=7)$. *: $\mathrm{p}<0.05$ compared to pretreatment.

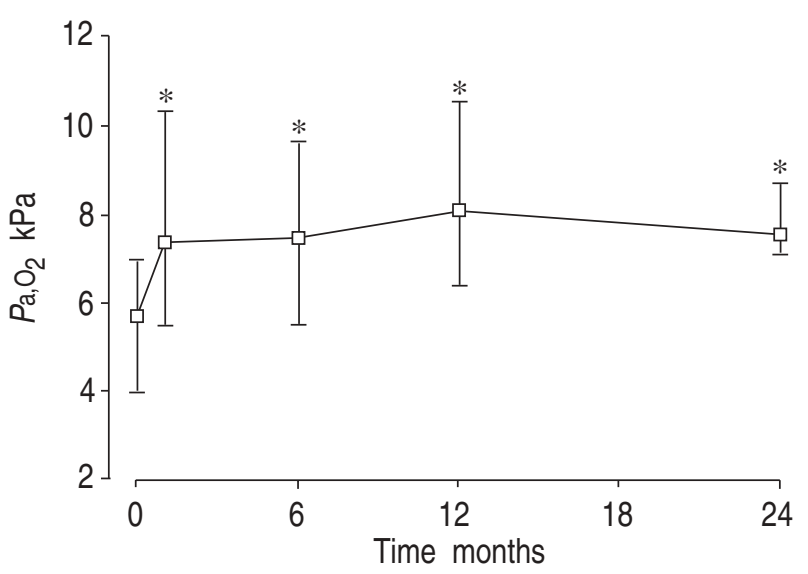

Fig. 3. - Median values and range in arterial oxygen tensions $\left(P_{\mathrm{a}, \mathrm{O}_{2}}\right)$ in patients treated with mask intermittent positive pressure ventilation at: pretreatment $(n=26) ; 1$ month $(n=20) ; 6$ months $(n=19) ; 1$ yr $(n=18)$; and 2 yrs $(n=7)$. * $p<0.05$ compared to pretreatment.

overall diuretic use for the population was similar at each time point.

Quality of life assessed by the SF-36 questionnaire revealed no significant changes in the patients' perceptions 


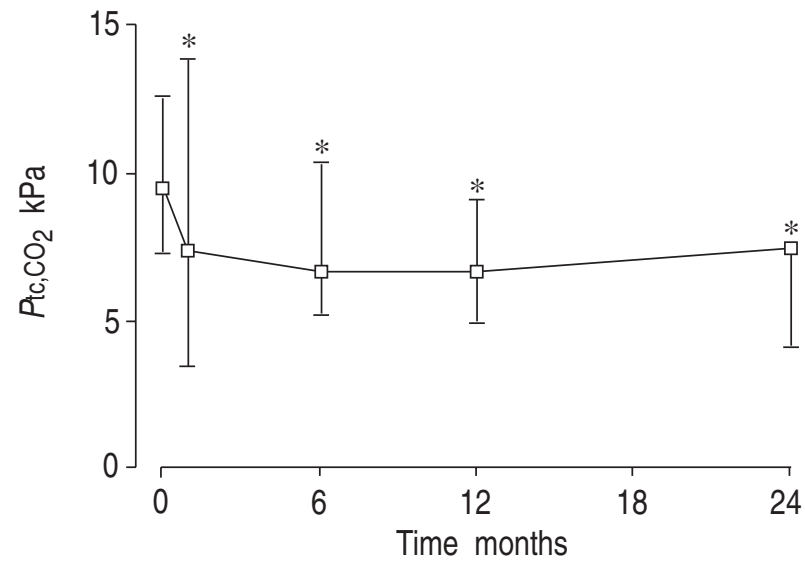

Fig. 4. - Median values and range in overnight transcutaneous carbon dioxide tensions $\left(P \mathrm{tc}, \mathrm{CO}_{2}\right)$ in patients treated with mask intermittent positive pressure ventilation at: pretreatment $(n=26) ; 1$ month $(n=20) ; 6$ months $(n=19) ; 1 \mathrm{yr}(n=18)$; and 2 yrs $(n=7)$. *: $p<0.05$ compared to pretreatment.

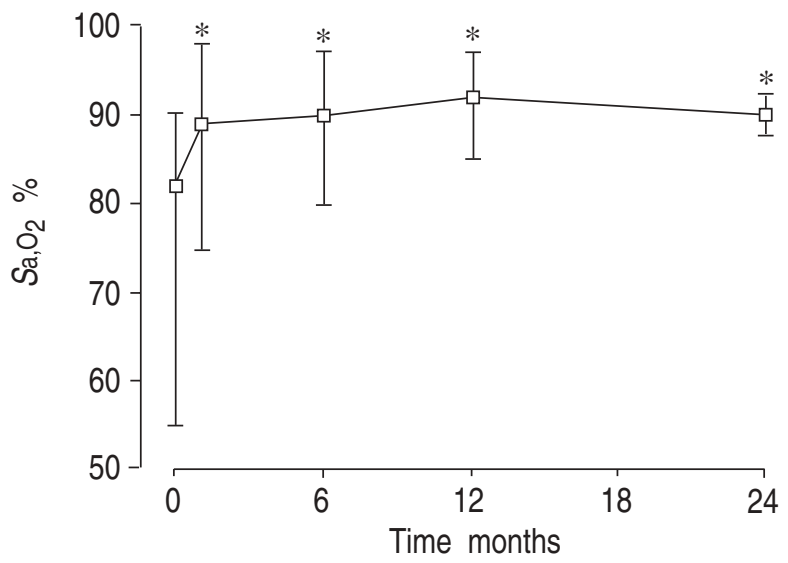

Fig. 5. - Median values and range in overnight arterial oxygen saturations $\left(\mathrm{S}_{\mathrm{a}, \mathrm{O}_{2}}\right)$ in patients treated with mask intermittent positive pressure ventilation at: pretreatment $(n=26) ; 1$ month $(n=20) ; 6$ months $(n=19) ; 1$ yr $(n=18)$; and 2 yrs $(n=7)$. *: $p<0.05$ compared to pretreatment.

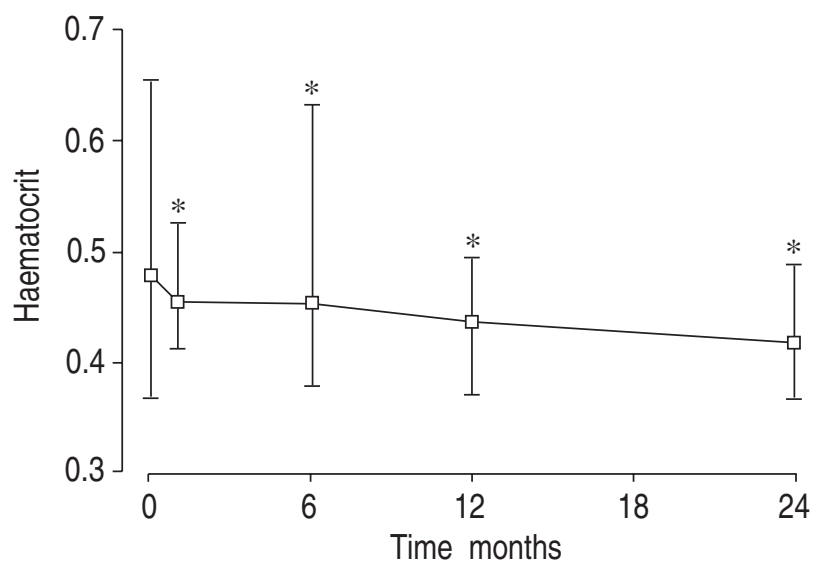

Fig. 6. - Median values and range of the haematocrit in patients treated with mask intermittent positive pressure ventilation at: pretreatment $(n=26) ; 1$ month $(n=20) ; 6$ months $(n=19) ; 1 \mathrm{yr}(n=18)$; and 2 yrs $(n=7)$. *: $\mathrm{p}<0.05$ compared to pretreatment.

of health in the domains of physical functioning, bodily pain, general health perceptions, energy, social functioning, role limitation because of emotional problems and mental health at pretreatment baseline compared to 1 and 6 months and 1 yr. Despite no perceived change in phys-

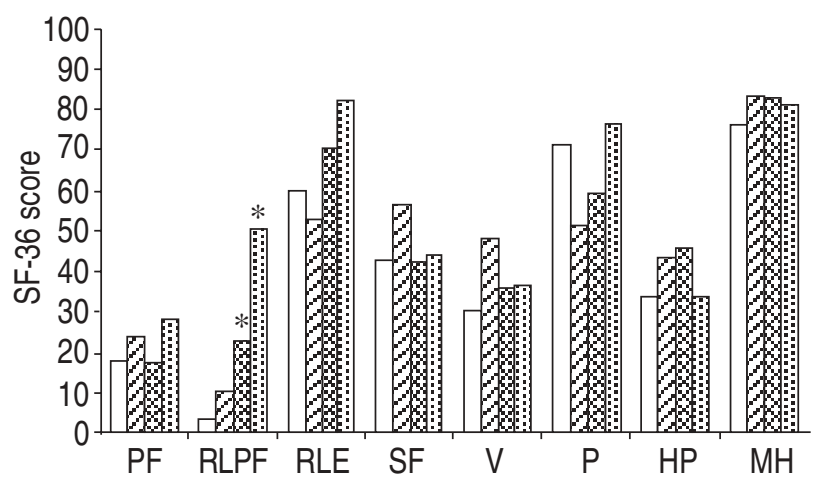

Fig. 7. - Median values of the SF-36 scores for 11 patients assessed at: $\square$ : pretreatment; $Q 2$ : 1 month; $\$$ : 6 months; 1 : 1 yr. PF: physical function; RLPF: role limitation due to physical function; RLE: role limitation due to emotional function; SF: social function; V: vitality; P: bodily pain; HP: health perception; MH: mental health. *: $\mathrm{p}<0.05 \mathrm{com}-$ pared to pretreatment.

cal function there was a significant improvement in role limitation due to physical problems before and after 6 months and $1 \mathrm{yr}$ of treatment. The overall changes in the median values for the above parameters are shown in figure 7.

\section{Discussion}

MIPPV is a well-established treatment for restrictive disorders $[14,15]$, but its role in the long-term treatment of COPD is not settled, with few and conflicting data with regard to survival, physiological changes and quality of life [8]. The present retrospective study of 26 patients was not controlled but the group is comparable to patients in several other studies of LTOT, tracheostomy ventilation and mask ventilation $[6,7,9,11,13,16,17]$. All the patients in the present study were hypoxic $\left(\mathrm{Pa}, \mathrm{O}_{2}<7.3 \mathrm{kPa}\right)$, had severe airflow obstruction, were in a stable clinical state prior to therapy and were aged $\breve{S} 35$ yrs. The selection criteria for the present study resulted in a higher mean $\mathrm{Pa}_{\mathrm{a}} \mathrm{CO}_{2}$ at entry and a lower FEV $1 \%$ pred in patients than in previous trials of LTOT, home mechanical ventilation via tracheostomy or mask ventilation, where data are available (table 3).

In the present study of 26 patients, none of whom could tolerate oxygen therapy alone but were treated with MIPPV \pm supplementary oxygen therapy, the 3 and $5 \mathrm{yr}$ survival rate was greater than the Medical Research Council (MRC) control group, the $15 \mathrm{~h} \cdot$ day $^{-1}$ oxygen treatment group in the MRC trial and the Association Nationale pour le Traitement à Domicile de l'Insufficience Respiratoire Chronique (ANTADIR) study "COPD" patients [9, 11]. The 3 yr survival was similar to the continuous oxygen therapy group in the Nocturnal Oxygen Therapy Trial (NOTT) study (table 3) [10]. The benefits on survival were probably even greater since eight patients in the present study had been on LTOT prior to starting MIPPV and would have already utilized some of the survival benefit that LTOT offered. These results suggest that in this selected group of patients with hypercapnic respiratory failure, MIPPV \pm supplementary oxygen is at least as effective as continuous oxygen therapy in improving survival rates.

Adequate oxygenation was achieved in our patients with MIPPV alone or with low oxygen flow rates. In $86 \%$ 
Table 3. - A comparison between the present study and previous studies using oxygen, tracheostomy or mask ventilation

\begin{tabular}{|c|c|c|c|c|c|c|c|c|c|c|c|}
\hline & \multirow[t]{3}{*}{ Year } & \multirow{3}{*}{$\begin{array}{l}\text { Sub- } \\
\text { jects } \\
n\end{array}$} & \multirow{3}{*}{$\begin{array}{c}\text { Acute } \\
\text { cases } \\
\text { included? }\end{array}$} & \multirow{3}{*}{$\begin{array}{l}\text { Age } \\
\text { yrs }\end{array}$} & \multirow{3}{*}{$\begin{array}{c}\mathrm{Pa}_{\mathrm{a}, \mathrm{CO}_{2}} \\
\mathrm{kPa}\end{array}$} & \multirow{3}{*}{$\begin{array}{l}P_{\mathrm{a}, \mathrm{O}_{2}} \\
\mathrm{kPa}\end{array}$} & \multirow{3}{*}{$\begin{array}{c}\text { FEV1 } \\
\text { L }\end{array}$} & \multirow{3}{*}{$\begin{array}{c}\text { FEV } 1 \\
\%\end{array}$} & \multicolumn{3}{|c|}{ Survival } \\
\hline & & & & & & & & & $1 \mathrm{yr}$ & $3 \mathrm{yrs}$ & $5 \mathrm{yrs}$ \\
\hline & & & & & & & & & $\mathrm{n}$ & $\mathrm{n}$ & $\mathrm{n}$ \\
\hline Present study & 1997 & 26 & No & 64.7 & 8.61 & 6.00 & 0.68 & 27.2 & 92 & 68 & 68 \\
\hline \multicolumn{12}{|l|}{ Oxygen therapy } \\
\hline MRC (control males) [9] & 1981 & 33 & No & 56.2 & 7.07 & 6.85 & 0.65 & - & 78 & 42 & 18 \\
\hline MRC (treated males) [9] & 1981 & 33 & No & 58.2 & 7.30 & 6.7 & 0.76 & - & 88 & 52 & 44 \\
\hline NOTT (nocturnal oxygen) [10] & 1980 & 102 & No & 65.7 & 5.77 & 6.85 & - & 29.5 & 80 & 44 & - \\
\hline NOTT (continuous therapy) [10] & 1980 & 101 & No & 65.2 & 5.77 & 6.76 & - & 29.5 & 87 & 66 & - \\
\hline ANTADIR $^{+}[9]$ & 1996 & 7370 & Yes & 68.2 & $6.22 *$ & $7.12 *$ & - & 35.3 & 74 & 47 & 30 \\
\hline \multicolumn{12}{|l|}{ Tracheostomy ventilation } \\
\hline ROBERT et al. [16] & 1983 & 50 & Yes & 60 & $5.85 *$ & $7.05^{*}$ & 0.72 & 33 & 88 & 54 & 18 \\
\hline Murr et al. [17] & 1994 & 259 & Yes & 63.3 & $7.44 *$ & $6.70 *$ & 0.73 & - & 88 & 60 & 44 \\
\hline \multicolumn{12}{|l|}{ Mask ventilation } \\
\hline LEGER et al. $[6]^{++}$ & 1994 & 50 & Yes & 63 & $7.05 *$ & $6.26^{*}$ & - & 36 & 85 & 75 & 43 \\
\hline Simonds et al. $[7]^{++}$ & 1995 & 33 & Yes & 57.4 & $8.3^{*}$ & $6.10 *$ & 0.58 & - & 88 & 70 & 43 \\
\hline
\end{tabular}

Values are presented as absolute value or as mean. +In the ANTADIR study $94.9 \%$ of COPD patients were treated with oxygen therapy alone although $3.0 \%$ and $2.1 \%$ were treated with mask or tracheostomy ventilation, respectively [13]. In the ANTADIR study the "COPD" group included patients with bronchiectasis and asthma and these have been excluded from comparison; ++: survival figures are those patients continuing ventilation; *: these results included patients with an acute exacerbation in whom these values may be worse than their stable state. MRC: Medical Research Council; NOTT: Nocturnal Oxygen Therapy Trial; ANTADIR: Association Nationale pour le Traitement à Domicile de l'Insufficience Respiratoire Chronique. For further defintions, see legend to table 1.

of patients the oxygen flow rates prescribed was $ð 1 \mathrm{~L}$. $\min ^{-1}$. The flow rates were individually titrated to achieve $\mathrm{Sa}, \mathrm{O}_{2}>90 \%$ once $\mathrm{Pa}_{\mathrm{a}} \mathrm{CO}_{2}$ was reduced. In contrast, patients in the MRC trial were treated with a uniform oxygen flow rate of $2 \mathrm{~L} \cdot \mathrm{min}^{-1}$ and in the NOTT study the minimum oxygen flow rate was $1 \mathrm{~L} \cdot \mathrm{min}^{-1}$ during the day and 2 $\mathrm{L} \cdot \mathrm{min}^{-1}$ at night $[9,10]$. The prescription of low oxygen flow rates in our patients minimized the risk of hypercapnia developing.

Survival comparisons between the patients in the present study treated with MIPPV \pm oxygen therapy and those in other studies using mechanical ventilation via a mask or tracheostomy, or oxygen therapy alone are complex because patients can start treatment at differing degrees of severity and stages in the natural history of their disease. In previous large series of the use of tracheostomy or MIPPV in COPD, the populations have been heterogeneous with a range of severity of airflow obstruction, $\mathrm{Pa}, \mathrm{O}_{2}$ and $\mathrm{Pa}_{\mathrm{a}} \mathrm{CO}_{2}$ (table 3) $[6,7,16,17]$. In some studies patients started on treatment during an acute deterioration $[6,17]$ or while transferring from naso- or orotracheal ventilation to tracheostomy or MIPPV [7, 17] have been included. The inclusion of acute cases is a confounding factor possibly resulting in an apparent improved long-term survival in these series. Although the hospital survival of patients with an acute exacerbation is poor, the survival of those who reach discharge from hospital without home ventilation was 86 and $91 \%$ at $1 \mathrm{yr}$ in two large studies $[18,19]$ which is superior to stable patients treated with LTOT. In our study, survival was better than the tracheostomy series with none of the morbidity associated with that treatment. Compared with other MIPPV series, results from the present study were worse at 3 yrs, probably due to our exclusion of acute cases, but despite this, the $5 \mathrm{yr}$ survival was superior to previously reported studies.

The predictors of poor survival in the present study were a low FEV1, a low BMI, a long duration of initial hospital stay and a high overnight $P \mathrm{tc}, \mathrm{CO}_{2}$. Our finding of an association between low BMI or body weight with poor survival agrees with other studies [10, 20, 21]. A low BMI has been correlated in a study of 126 patients with stable COPD with a low FEV1 suggesting that they are not independent predictors of poor survival [22]. A high daytime $\mathrm{Pa}, \mathrm{CO}_{2}$ has been shown to be a predictor of poor survival either with $[9,23]$ or without $[12,13]$ oxygen therapy. In the ANTADIR study "COPD" patients with a high $\mathrm{Pa}, \mathrm{CO}_{2}$ had a high initial mortality but after $1 \mathrm{yr}$ had a greater survival chance than those with a low or normal $P \mathrm{a}, \mathrm{CO}_{2}$. However the "COPD" patient group in this study included subjects with asthma or bronchiectasis, and $15 \%$ of patients had a $\mathrm{Pa}, \mathrm{O}_{2}>8 \mathrm{kPa}$ [11].

In the present study, $\mathrm{Pa}, \mathrm{CO}_{2}$ was not found to be of prognostic value partly as result of entry criteria which excluded patients with a low or normal $\mathrm{Pa}_{\mathrm{a}} \mathrm{CO}_{2}$. More importantly, the initiation of MIPPV reduced $\mathrm{Pa}_{2}, \mathrm{CO}_{2}$ by 1.8 $\mathrm{kPa}$ in patients thus eliminating or ameliorating this risk factor for mortality.

Other studies of MIPPV in COPD have shown that it can reduce $P \mathrm{a}, \mathrm{CO}_{2}$ and increase $P \mathrm{a}, \mathrm{O}_{2}[2,6,7]$ though in one series it had no effect [5]. In the current study, a rise is also shown in the median daytime $\mathrm{Pa}, \mathrm{O}_{2}$ of $2.4 \mathrm{kPa}$ and nocturnal $\mathrm{S}_{\mathrm{a}} \mathrm{O}_{2}$ (excluding patients started on oxygen). The differences in daytime arterial blood gas measurement were significant after only 1 month and were maintained thereafter.

The significant decrease in haematocrit noted after treat-ment with MIPPV \pm oxygen therapy probably reflects improved oxygenation and subsequent reduction in secondary polycythaemia. This could not be explained by an alteration in the plasma volume caused by diuretic thera-py as there was no overall change in diuretic therapy for the study population. The continued reduction up to 1 yr may be attributable to the gradual increase in the median $\mathrm{Pa}_{\mathrm{a}} \mathrm{O}_{2}$ up to $1 \mathrm{yr}$ and the delayed haematological 
response caused by the life span of the erythrocyte which is $100-120$ days.

All patients continued to comply with MIPPV, with $81 \%$ using it for at least $7 \mathrm{~h} \cdot$ day $^{-1}$. Other studies of MIPPV in COPD have emphasized difficulties tolerating the mask, abdominal distension, poor sleep and anxiety, which usually arise within 3 months of starting ventilation $[1,4,5]$. In two large long-term studies of MIPPV in COPD 6 and $15 \%$ of patients discontinued therapy $[6,7]$. The superior results in the present study might be due to a longer inpatient initiation of therapy: patients were only discharged when they had fully adjusted to using MIPPV. This took between 6 and 14 days compared to 2 and 5 days allocated in the study by LEger et al. [6]. Domiciliary visits were available to patients who lived within a 30 mile radius of the hospital. All patients had regular in-patient review with the option of using a $24 \mathrm{~h}$ telephone helpline for any problems encounted with mask ventilation.

The only previous study of the SF-36 in patients with COPD on ventilation was a single estimation when patients were already on therapy [7]. Subjects in the present study were more incapacitated with a greater degree of physical impairment but had similar mental health, social function, bodily pain and energy scores (using pretreatment data). One previous study of the neuropsychological function and physical function of MIPPV in COPD using different tests revealed improved attention, verbal and visual memory, constructional apraxia and self regulation but no change in physical function [5]. The quality of life on MIPPV measured by the SF-36 in patients in the present study was unchanged except for the domain of role limitation by physical health which significantly improved at 6 months and $1 \mathrm{yr}$. This was derived from questions on the physical health of patients over the preceding 4 weeks concerning: 1) Had they cut down the amount of time spent on activities? 2) Had they accomplished less than they would have liked? 3) Were they limited in the activities they could perform? and 4) Had they experienced difficulty performing their work or other activities. The results suggest that patients treated with MIPPV show an acceptance of their severely impaired quality of life or perhaps an adaptation to it allowing them to circumvent this disability despite no change in their self-reported physical function.

It is recommend that patients with hypercapnic respiratory failure commenced on oxygen therapy should be assessed with arterial blood gas measurements, pulse oximetry and $P \mathrm{tc}, \mathrm{CO}_{2}$ during sleep both before and after therapy. If supplementary oxygen therapy fails to raise the daytime $\mathrm{Pa}, \mathrm{O}_{2}$ to $>7.3 \mathrm{kPa}$ without a rise in the awake $P \mathrm{a}, \mathrm{CO}_{2}$ to $>8 \mathrm{kPa}$ or raise nocturnal oxygen saturations to $>90 \%$ without a rise in the transcutaneous $P \mathrm{tc}, \mathrm{CO}_{2}$ to $>9$ $\mathrm{kPa}, \mathrm{MIPPV}$ should be instituted. Oxygen therapy may be required to supplement MIPPV if this alone fails to raise $P \mathrm{a}, \mathrm{O}_{2}$ despite a fall in $\mathrm{Pa}, \mathrm{CO}_{2}$.

The findings indicate that mask intermittent positive pressure ventilation in hypercapnic patients with chronic obstructive pulmonary disease unable to tolerate oxygen therapy is associated with a prognosis which is at least as good as eucapnic patients treated with long-term oxygen therapy and better than hypercapnic patients treated with long-term oxygen therapy and less well-defined groups treated with tracheostomy ventilation. The results were achieved with $86 \%$ of patients receiving $\partial 1 \mathrm{~L} \cdot \mathrm{min}^{-1}$ oxy- gen and all patients tolerating mask ventilation. The high compliance and the emphasis on attempting to normalize the arterial blood gases probably contributed to the prolonged survival in this series.

\section{References}

1. Carroll N, Branthwaite MA. Control of nocturnal hypoventilation by nasal intermittent positive pressure ventilation. Thorax 1988; 43: 349-353.

2. Elliot MW, Mulvey DA, Moxham J, Green M, Branthwaite MA. Domiciliary nocturnal nasal intermittent positive pressure ventilation in COPD: mechanisms underlying changes in arterial blood gas tension. Eur Respir J 1991: 4: 1044-1052.

3. Meecham Jones DJ, Wedzicha IA. Comparison of pressure and volume preset nasal ventilator systems in stable chronic respiratory failure. Eur Respir J 1993; 6: 10601064.

4. Elliot MW, Simonds AK, Carroll MP, Wedzicha JA, Branthwaite MA. Domiciliary nocturnal nasal intermittent pressure ventilation in hypercapnic respiratory failure due to chronic obstructive lung disease: effect on sleep and quality of life. Thorax 1992; 47: 342-348.

5. Strumpf DA, Millman RP, Carlisle CC, et al. Nocturnal positive pressure ventilation via nasal mask in patients with severe chronic obstructive pulmonary disease. Am Rev Respir Dis 1991; 144: 1234-1239.

6. Leger P, Bedicam JM, Cornette A, et al. Nasal intermittent positive pressure ventilation long term follow-up in patients with severe chronic respiratory insufficiency. Chest 1994; 105: 100-105.

7. Simonds AK, Elliot MW. Outcome of domiciliary nasal positive pressure ventilation in restrictive and obstructive disorders. Thorax 1995; 50: 604-609.

8. Shneerson JM. The changing role of mechanical ventilation in COPD. Eur Respir J 1996; 9: 393-398.

9. Stuart-Harris C, Bishop JM, Clark TJH, et al. Long term domiciliary oxygen therapy in chronic hypoxic cor pulmonale complicating chronic bronchitis and emphysema. Lancet 1981; (i): 681-686.

10. Nocturnal oxygen therapy trial group. Continuous or nocturnal oxygen therapy in hypoxemic chronic obstructive lung disease. Ann Intern Med 1980; 93: 391-398.

11. Chaileux E, Faroux B, Binet F, Dautzenberg B, Polu JM. Predictors of survival in patients receiving domiciliary oxygen therapy or mechanical ventilation. Chest 1996; 109: 741-749.

12. Postma DS, Burema J, Gimeno F, et al. Prognosis in severe chronic obstructive pulmonary disease. Am Rev Respir Dis 1979; 119: 357-367.

13. Travers GA, Cline MG, Burrows B. Predictors of mor-tality in chronic obstructive pulmonary disease. Am Rev Respir Dis 1979; 119: 895-902.

14. Ellis ER, Bye PTP, Bruderer JW, Sullivan CE. Treatment of respiratory failure during sleep in patients with neuromuscular disease. Am Rev Respir Dis 1987; 135: 148152.

15. Bach JR, Alba A, Mosher R, Delaubier A. Intermittent positive pressure ventilation via nasal access in the management of respiratory insufficiency. Chest 1987; 92: $168-170$.

16. Robert D, Gerard M, Leger P, et al. Domiciliary ventilation by tracheotomy for chronic respiratory failure. Rev Fr Mal Respir 1983; 11: 923-936.

17. Muir JF, Girault C, Cardinaud JP, Polu JM. Survival and 
long term follow-up of tracheostomised patients with COPD treated by home mechanical ventilation. Chest 1994; 106: 201-209.

18. Martin TR, Lewis SW, Albert RK. The prognosis of patients with chronic obstructive pulmonary disease after hospitalisation for acute respiratory failure. Chest 1982; 82: 310-314.

19. Seneff MG, Wagner DP, Wagner RP, Zimmerman JE, Knaus WA. Hospital and 1 year survival of patients admitted to intensive care units with acute exacerbation of chronic obstructive pulmonary disease. JAMA 1995; 274 : 1852-1857.

20. Boushy SF, Adhikiri PK, Sakamoto A, Lewis B. Factors affecting prognosis in emphysema. Chest 1964; 45: 407411.

21. Wilson DO, Rogers RM, Wright EC, Anthonisen NR. Body weight in chronic pulmonary disease: the NIH IPPB trial. Am Rev Respir Dis 1989; 139: 1435-1438.

22. Sahebjami H, Doers JT, Render ML, Bond TL. Anthropometric and pulmonary function test profiles of outpatients with stable chronic obstructive pulmonary disease. Am J Med 1993; 94: 469-474.

23. Cooper CB, Waterhouse J, Howard P. Twelve year clinical study of patients with hypoxic cor pulmonale given long term domiciliary oxygen therapy. Thorax 1987; 42: $105-110$. 